\title{
The anterior interhemispheric approach to a third ventricular cavernous malformation
}

\author{
Nikolay L. Martirosyan, MD, M. Yashar S. Kalani, MD, PhD, Peter Nakaji, MD, \\ and Robert F. Spetzler, MD
}

Department of Neurosurgery, Barrow Neurological Institute and St. Joseph's Hospital and Medical Center, Phoenix, Arizona

The anterior interhemispheric approach is a workhorse for treatment of lesions in the third ventricle. In this case, we demonstrate the utility of this approach for resecting a complex third ventricular cavernous malformation. We discuss patient positioning, optimal location of the craniotomy, and surgical resection techniques for safe removal of these lesions. We also demonstrate the importance of gravity retraction using the falx to prevent injury to the dominant frontal lobe.

The video can be found here: https://youtu.be/38woc28er7M.

KEYWORDS anterior interhemispheric approach; cavernous malformation; third ventricle; video 